\title{
Factors Associated With The Use of Rehabilitation Therapies Among Brazilian Adults With Chronic Diseases And Functional Limitations: A Cross- Seccional Study
}

\section{Patricia de Moraes Mello Boccolini}

Núcleo de Informação, Políticas Públicas e Inclusão Social - NIPPIS, FMP/UNIFASE, Petrópolis, Rio de Janeiro

Cristina Maria Rabelais Duarte

Núcleo de Informação, Políticas Públicas e Inclusão Social - NIPPIS, FMP/UNIFASE, Petrópolis, Rio de Janeiro

Cristiano Siqueira Boccolini ( $\square$ cristianoboccolini@gmail.com )

Instituto de Comunicação e Informação Científica e Tecnológica em Saúde, Fundação Oswaldo Cruz, Rio de Janeiro

\section{Research Article}

Keywords: rehabilitation, chronic diseases, national health survey

Posted Date: September 24th, 2021

DOI: https://doi.org/10.21203/rs.3.rs-901733/v1

License: (1) This work is licensed under a Creative Commons Attribution 4.0 International License. Read

Full License 


\section{Abstract}

Background: Understanding the patterns of rehabilitation therapies among individuals who need them in Brazil can help identify the most vulnerable populations and guide public policies. We aim to analyze the use of rehabilitation therapies among Brazilian adults with chronic conditions and limitations in their daily activities. Methods: This is a population-based survey with data from the 2013 National Health Survey, whose complex sample consisted of three-stage clusters. The outcome considered was the adult population who reported a stroke, arthritis, work-related musculoskeletal disorder (WMSD), chronic obstructive pulmonary disease (COPD), or reported back pain, associated with moderate to very intense limitations of their activities and who have used rehabilitation therapies. A logistic regression model was estimated, with a $95 \% \mathrm{Cl}$, adjusted for sociodemographic variables.

Results: Among Brazilian adults, 24.8\% were diagnosed with a stroke, arthritis, WMSD, COPD, or reported back pain. Among these, $34.6 \%$ reported having moderate, intense, or very intense limitations in their daily activities due to illnesses, and, among this population, $26.3 \%$ performed some rehabilitation therapy related to their conditions. The use of rehabilitation therapies was more frequent among the female population, with higher education, higher socioeconomic status, older, and living in the southern macro-region of Brazil.

Conclusions: the low frequency of rehabilitation therapies may be associated with barriers to access health services.

\section{Background}

The epidemiological and demographic transition observed in recent decades are associated with the increased prevalence of non-communicable chronic diseases, such as cardiovascular diseases, cancer, diabetes, and respiratory diseases, and with an increase in the functional limitation of individuals, especially in the elderly population ${ }^{1,2}$. According to the World Health Organization (WHO), $10 \%$ of the population in developed countries has some functional limitation, and this percentage increases to $15 \%$ in developing countries ${ }^{3}$.

The WHO conceptualizes rehabilitation as measures that help people with disabilities, or some transient or permanent functional limitation, to have better functionality in their interaction with their environment ${ }^{4}$. In this sense, rehabilitation can present two aspects: actions focusing on the individual, as indicated by the WHO, and equipment that can help in the rehabilitation process $^{5}$.

Rehabilitation services are a fundamental component in the health care for people with chronic diseases and functional limitations, providing to this population therapies to recovery, support for social inclusion, and improvement in the quality of life ${ }^{6}$. In Brazil, there is a health care network for people with chronic diseases, funded by the Unified Health System (SUS), that should guarantee rehabilitation services to all users with chronic diseases to meet their needs ${ }^{6}$.

Understanding the patterns of rehabilitation therapies among individuals who potentially need them in Brazil can help identify the most vulnerable populations and guide public health policies. Therefore, we aimed to 
analyze the factors associated with rehabilitation therapies among Brazilian adults with chronic diseases and limitations in their daily activities.

\section{Methods}

\section{Study design, objective, and sampling}

This study had a cross-sectional design with data from the National Health Survey (PNS), an epidemiological survey with a household basis and national representation, conducted in 2013 by the Oswaldo Cruz Foundation and the Ministry of Health in partnership with the Brazilian Institute of Geography and Statistics (IBGE).

The study aimed to analyze the factors associated with rehabilitation therapies among Brazilian adults with chronic diseases and limitations in their usual activities.

The sample consisted of clusters in three stages: selection of Primary Analysis Units (UPAs), followed by random selection of households (fixed number per UPAs), and, finally, in each selected household, an adult resident was randomly selected (out of 18 years of age or older). The survey sample comprised 81,254 households, of which 69,994 occupied. In the end, 64,348 household interviews and 60,202 were conducted with the selected resident 7,8 .

\section{Outcome and associated variables}

The outcome evaluated in this study was the use of rehabilitation therapies in individuals who reported a diagnosis of the following chronic diseases: ischemic stroke or stroke; arthritis or rheumatism, back pain (chronic spine problem, such as chronic back pain or in the neck, low back pain, sciatica, problems in the vertebrae or disc), Work-related Musculoskeletal Disorder (WMSD) and Chronic Obstructive Pulmonary Disease (COPD), and who also reported moderate to severe limitations in their daily activities. The PNS-2013 had questions about rehabilitation only for respondents that reported those specific diseases. Since WMSDs includes several disorders directly related to work, which can affect different parts of the body, including the spine, the PNS questionnaire addresses, separately, WMSD and spinal problems, which refer to the presence of "chronic spine pain or disorders, such as chronic back or neck pain, low back pain, sciatica, vertebrae or disc diseases." When referring to WMSD, the PNS-2013 asked the question, "has a doctor already given you the diagnosis of WMSD? ${ }^{9}$

For each type of chronic disease included in this study, the subject reported using or not a different rehabilitation set. For stroke, it was asked whether the individual had used physiotherapy or other rehabilitation therapies. For arthritis, the therapies included were the practice of exercise or some physical activity, in addition to physiotherapy and acupuncture. For back pain and WMSD, it was asked about the use of acupuncture, or exercise, or physiotherapy. The use of medications was not considered a rehabilitation therapy, and no distinction was made between physical activity guided or accompanied by a health professional and spontaneous activity or leisure. 
The sociodemographic characteristics associated with the outcome: Brazilian region of residence (Southeast, South, Midwest, Northeast, North); sex (male; female); age group (18-39; 40-59; and 60 years and over); level of education (incomplete elementary school; complete high school and more); skin color/race (white; nonwhite); and socioeconomic level10, divided into classes $A+B, C, D+E^{10}$.

In all statistical analyses, we incorporated the complex sample design. Initially, with a 95\% confidence interval, we described the prevalence of chronic diseases and conditions, limitations of moderate, intense, and very intense activities, and the use of rehabilitation therapies according to disease or chronic condition. Next, we analyzed the prevalence, with a $95 \%$ confidence interval, of moderate, intense, and very intense limitations, according to disease or chronic condition and sociodemographic characteristics, and among these, the proportion of those who used rehabilitation therapies.

Finally, we estimated the chance of using rehabilitation therapies, according to disease or chronic condition and sociodemographic variables, by calculating the unadjusted odds ratio (OR) and adjusted odds ratio (AOR), obtained by the logistic regression model.

For each sociodemographic variable, the ORs using the following response categories as a reference for comparison: male gender, non-white race/skin color, incomplete primary education, age between 18 and 39 years, and the Northeast region of the country. Values equivalent to one were considered non-significant, with a $95 \%$ confidence interval.

\section{Results}

Table 1 shows the prevalence of self-reported medical diagnosis of chronic diseases and the consequent limitations of moderate, intense, and very intense daily activities. Back pain and arthritis are the most prevalent diseases, and stroke and lung disease were the least prevalent. Regarding the limitation of daily activities, stroke and arthritis were the diseases that more frequently limited daily activities in a moderate, intense, and very intense way. About $25 \%$ of subjects reported a diagnosis of one or more of the study diseases. Among them, the prevalence of moderate, intense, and very intense limitation of daily activities was about one-third. 
Table 1

Prevalence of chronic diseases and limitation of daily activities among the Brazilian adult population. PNS, Brazil, 2013.

\begin{tabular}{|c|c|c|c|c|}
\hline \multirow{2}{*}{$\begin{array}{l}\text { Chronic disease or } \\
\text { condition }^{1}\end{array}$} & \multirow{2}{*}{$\begin{array}{l}\text { Prevalence }{ }^{2} \\
(\%)\end{array}$} & \multirow[t]{2}{*}{ Cl $95 \%$} & \multicolumn{2}{|c|}{ Limitation of daily activities ${ }^{3}$} \\
\hline & & & Prevalence (\%) & Cl $95 \%$ \\
\hline $\mathrm{CVA}^{4}$ & 1.5 & $(1.4-1.7)$ & 38.6 & $\begin{array}{l}(34.7- \\
42.6)\end{array}$ \\
\hline Arthritis 5 & 6.4 & $(6.1-6.8)$ & 34.9 & $\begin{array}{l}(32.4- \\
37.5)\end{array}$ \\
\hline Back Pain 6 & 18.5 & $\begin{array}{l}(17.8- \\
19.1)\end{array}$ & 34.7 & $\begin{array}{l}(33.2- \\
36.3)\end{array}$ \\
\hline WMSD $^{7}$ & 2.4 & $(2.2-2.7)$ & 30.3 & $\begin{array}{l}(26.7- \\
34.1)\end{array}$ \\
\hline COPD $^{8}$ & 1.8 & $(1.6-2.0)$ & 24.0 & $\begin{array}{l}(20.7- \\
27.6)\end{array}$ \\
\hline One or more conditions ${ }^{9}$ & 24.8 & $\begin{array}{l}(24.1- \\
25.5)\end{array}$ & 34.6 & $\begin{array}{l}(33.3- \\
35.9)\end{array}$ \\
\hline Total & 100.0 & & & \\
\hline \multicolumn{5}{|c|}{$\begin{array}{l}\text { Chronic disease or condition }{ }^{1} \text { : Self-reported medical diagnosis of disease or condition; Prevalence }{ }^{2} \text { : taking } \\
\text { into account the complex design of the sample. } 95 \% \text { Cl: } 95 \% \text { confidence interval taking into account the } \\
\text { complex design of the sample. Limitation of their daily activities }{ }^{3} \text { such as: working, studying, and doing } \\
\text { housework (report of moderate, intense, or very intense limitations of chronic diseases). Cerebrovascular } \\
\text { accident (CVA), ischemic stroke or stroke }{ }^{4} \text {; Arthritis or rheumatism }{ }^{5} \text {; Back Pain: Chronic back problem } \\
\text { such as chronic back or neck pain, low back pain, sciatica, vertebrae or disc problems }{ }^{6} ; \text { WMSD: work- } \\
\text { related musculoskeletal disorder }{ }^{7} \text {; COPD: Chronic Obstructive Pulmonary Disease }{ }^{8} \text {. One or more } \\
\text { conditions: report of one or more chronic diseases or conditions }{ }^{9} \text {. }\end{array}$} \\
\hline
\end{tabular}

Table 2 shows the prevalence of individuals who reported using one or more rehabilitation therapies due to the selected chronic diseases and reported limitations in their daily activities. Individuals who reported having arthritis and WMSD were those who most used rehabilitation therapies. Exercise or some physical activity was the most used therapy among individuals who reported having arthritis, and exercise, physical therapy, or both were the most used among those who reported having WMSD. Among the individuals who reported having COPD and limited their usual activities, they were the ones who least used rehabilitation therapies. 
Table 2

Prevalence of the use of therapies for chronic diseases among adults with moderate, intense, or very intense limitations of daily activities. PNS, Brazil, 2013.

\section{Therapies}

Prevalence $^{1} \quad \mathrm{Cl} 95 \%{ }^{2}$

(\%)

\section{CVA}

Physiotherapy

29.2

$(25.2-$

Other Rehabilitation Therapies

13.4

(9.0-19.5)

Reported the use of rehabilitation therapies due to CVA

30.2

(26.1-

34.5)

\section{Arthritis}

Exercise or some physical activity

23.6

(20.3-

27.3)

Physiotherapy

22.0

$(19.0-25.3)$

Acupuncture

4.3

$(3.2-5.6)$

Reported the use of rehabilitation therapies due to arthritis

36.5

(32.840.3)

\section{Back pain}

Exercise or physiotherapy

19.2

(17.3-

Acupuncture

3.0

$(2.3-3.8)$

Reported the use of rehabilitation therapies due for back pain

20.0

(18.0-

22.0)

WMSD

Exercise or physiotherapy

32.7

(28.3-

37.5)

Acupuncture

3.3

$(2.0-5.4)$

Reported the use of rehabilitation therapies due to WMSD

34.0

(29.3-

39.0)

COPD

Respiratory fisioterapy

10.0

$(8.6-11.6)$

Reported the use of rehabilitation therapies due to any of the selected
chronic diseases (total)

(24.4-

28.2)

Prevalence: taking into account the complex design of the sample ${ }^{1} .95 \% \mathrm{Cl}: 95 \%$ confidence interval taking into account the complex design of the sample ${ }^{2}$. 
Table 3 shows the sociodemographic characteristics of individuals with a chronic disease and reported moderate, intense, and very intense limitations in their daily activities. Chronic diseases were more frequent among women than men, non-white race, incomplete elementary education, Social Score C, between 40 and 59 years old, and the Southeast region. Stroke was more prevalent among men than women. COPD was more frequent among individuals who reported white than non-white race. Stroke, arthritis, and COPD were more prevalent among older than younger individuals, whereas WMSD and back pain was more prevalent among individuals between 40 and 59 years. 
Table 3

Prevalence of moderate, intense or very intense limitations in daily activities among subjects with chronic diseases, by sociodemographic characteristics. PNS, Brazil, 2013.

\begin{tabular}{|c|c|c|c|c|c|c|}
\hline \multirow[t]{3}{*}{ Variables } & CVA & Arthritis & Back pain & WMSD & COPD & Total $^{1}$ \\
\hline & $\%$ & $\%$ & $\%$ & $\%$ & $\%$ & $\%$ \\
\hline & (Cl 95\%) & (Cl 95\%) & (Cl 95\%) & (Cl 95\%) & (Cl 95\%) & (Cl 95\%) \\
\hline \multicolumn{7}{|l|}{ Sex } \\
\hline \multirow[t]{2}{*}{ Men } & 54.6 & 24.5 & 37.6 & 25.1 & 44.8 & 37.4 \\
\hline & $\begin{array}{l}(47.1- \\
61.9)\end{array}$ & $\begin{array}{l}(21.4- \\
27.9)\end{array}$ & $\begin{array}{l}(35.5- \\
39.9)\end{array}$ & $\begin{array}{l}(20.3- \\
30.6)\end{array}$ & $\begin{array}{l}(41.3- \\
48.4)\end{array}$ & $\begin{array}{l}(35.4- \\
39.5)\end{array}$ \\
\hline \multirow[t]{2}{*}{ Women } & 45.4 & 75.5 & 62.4 & 74.9 & 55.2 & 62.6 \\
\hline & $\begin{array}{l}(38.1- \\
52.9)\end{array}$ & $\begin{array}{l}(72.1- \\
78.6)\end{array}$ & $\begin{array}{l}(60.1- \\
64.5)\end{array}$ & $\begin{array}{l}(69.4- \\
79.7)\end{array}$ & $\begin{array}{l}(51.6- \\
58.7)\end{array}$ & $\begin{array}{l}(60.5- \\
64.6)\end{array}$ \\
\hline \multicolumn{7}{|l|}{ Race $^{2}$} \\
\hline \multirow[t]{2}{*}{ White } & 47.3 & 50.7 & 47.8 & 47.2 & 54.1 & 47.8 \\
\hline & $\begin{array}{l}(42.7- \\
52.0)\end{array}$ & $\begin{array}{l}(47.0- \\
54.5)\end{array}$ & $\begin{array}{l}(45.5- \\
50.1)\end{array}$ & $\begin{array}{l}(41.5- \\
52.8)\end{array}$ & $\begin{array}{l}(45.8- \\
62.3)\end{array}$ & $\begin{array}{l}(45.7- \\
49.9)\end{array}$ \\
\hline \multirow[t]{2}{*}{ Non-white } & 52.7 & 49.3 & 52.2 & 52.8 & 45.9 & 52.2 \\
\hline & $\begin{array}{l}(48.0- \\
57.3)\end{array}$ & $\begin{array}{l}(45.5- \\
53.0)\end{array}$ & $\begin{array}{l}(49.9- \\
54.5)\end{array}$ & $\begin{array}{l}(47.2- \\
58.8)\end{array}$ & $\begin{array}{l}(37.7- \\
54.2)\end{array}$ & $\begin{array}{l}(50.1- \\
54.3)\end{array}$ \\
\hline \multicolumn{7}{|l|}{ Education $^{3}$} \\
\hline \multirow{2}{*}{$\begin{array}{l}\text { Elementary } \\
\text { incomplete }\end{array}$} & 61.3 & 61.4 & 63.2 & 46.3 & 56.1 & 60.7 \\
\hline & $\begin{array}{l}(56.9- \\
65.5)\end{array}$ & $\begin{array}{l}(57.7- \\
65.0)\end{array}$ & $\begin{array}{l}(60.8- \\
65.6)\end{array}$ & $\begin{array}{l}(41.1- \\
51.5)\end{array}$ & $\begin{array}{l}(49.8- \\
62.2)\end{array}$ & $\begin{array}{l}(58.5- \\
62.8)\end{array}$ \\
\hline \multirow[t]{2}{*}{ High School } & 29.3 & 28.2 & 27.1 & 43.4 & 30.5 & 28.9 \\
\hline & $\begin{array}{l}(22.5- \\
37.1)\end{array}$ & $\begin{array}{l}(24.8- \\
31.8)\end{array}$ & $\begin{array}{l}(24.8- \\
29.5)\end{array}$ & $\begin{array}{l}(38.2- \\
48.6)\end{array}$ & $\begin{array}{l}(25.3- \\
36.2)\end{array}$ & $\begin{array}{l}(26.9- \\
31.1)\end{array}$ \\
\hline Higher education & 9.5 & 10.4 & 9.7 & 10.4 & 13.4 & 10.4 \\
\hline
\end{tabular}

Total ${ }^{1}$ : individuals with at least one of the selected diseases and limitations due to one of these diseases. Race: obtained by self-classification according to the IBGE categories; later categorized as white and nonwhite (black, brown, yellow, or indigenous) ${ }^{2}$. Education: categorized into incomplete elementary school (no education or incomplete elementary school), high school (complete high school and incomplete college) and higher education (complete college or more) ${ }^{3}$. Social score: classification proposed by the Brazilian Association of Population Studies (http://www.abep.org/criterio-brasil), where the population is divided into five categories, ranging from $A$ (richest) to $E$ (poorest) ${ }^{4}$. 


\begin{tabular}{|c|c|c|c|c|c|c|}
\hline \multirow[t]{4}{*}{ Variables } & CVA & Arthritis & Back pain & WMSD & COPD & Total $^{1}$ \\
\hline & $\%$ & $\%$ & $\%$ & $\%$ & $\%$ & $\%$ \\
\hline & (Cl 95\%) & (Cl 95\%) & (Cl 95\%) & (Cl 95\%) & (Cl 95\%) & (Cl 95\%) \\
\hline & $(5.4-16.2)$ & $(7.7-13.9)$ & $(8.1-11.4)$ & $(8.0-13.2)$ & $\begin{array}{l}(10.6- \\
16.8)\end{array}$ & $(8.9-12.0)$ \\
\hline \multicolumn{7}{|l|}{ Social Score ${ }^{4}$} \\
\hline \multirow[t]{2}{*}{$A+B$} & 15.8 & 19.7 & 22.1 & 33.7 & 33.5 & 22.9 \\
\hline & $\begin{array}{l}(11.8- \\
20.9)\end{array}$ & $\begin{array}{l}(16.3- \\
23.6)\end{array}$ & $\begin{array}{l}(19.9- \\
24.6)\end{array}$ & $\begin{array}{l}(29.4- \\
38.3)\end{array}$ & $\begin{array}{l}(27.5- \\
40.1)\end{array}$ & $\begin{array}{l}(20.9- \\
25.0)\end{array}$ \\
\hline \multirow[t]{2}{*}{ C } & 45.3 & 48.0 & 41.8 & 50.1 & 35.4 & 42.9 \\
\hline & $\begin{array}{l}(41.2- \\
49.4)\end{array}$ & $\begin{array}{l}(44.2- \\
51.8)\end{array}$ & $\begin{array}{l}(39.3- \\
44.4)\end{array}$ & $\begin{array}{l}(45.0- \\
55.2)\end{array}$ & $\begin{array}{l}(29.7- \\
41.5)\end{array}$ & $\begin{array}{l}(40.7- \\
45.1)\end{array}$ \\
\hline \multirow[t]{2}{*}{$D+E$} & 38.9 & 32.3 & 36.1 & 16.2 & 31.1 & 34.2 \\
\hline & $\begin{array}{l}(34.0- \\
44.0)\end{array}$ & $\begin{array}{l}(29.8- \\
34.9)\end{array}$ & $\begin{array}{l}(33.9- \\
38.3)\end{array}$ & $\begin{array}{l}(12.6- \\
20.6)\end{array}$ & $\begin{array}{l}(28.1- \\
34.3)\end{array}$ & $\begin{array}{l}(32.4- \\
36.1)\end{array}$ \\
\hline \multicolumn{7}{|l|}{ Age group } \\
\hline \multirow[t]{2}{*}{18 to 39 years old } & 2.0 & 6.1 & 18.3 & 26.5 & 18.5 & 17.2 \\
\hline & $(1.1-3.5)$ & $(4.9-7.6)$ & $\begin{array}{l}(16.6- \\
20.0)\end{array}$ & $\begin{array}{l}(21.7- \\
31.9)\end{array}$ & $\begin{array}{l}(13.7- \\
24.6)\end{array}$ & $\begin{array}{l}(15.7- \\
18.9)\end{array}$ \\
\hline \multirow[t]{2}{*}{40 to 59 years old } & 33.5 & 45.1 & 50.1 & 62.9 & 30.5 & 47.8 \\
\hline & $\begin{array}{l}(29.5- \\
37.7)\end{array}$ & $\begin{array}{l}(41.2- \\
49.0)\end{array}$ & $\begin{array}{l}(47.8- \\
52.4)\end{array}$ & $\begin{array}{l}(57.3- \\
68.1)\end{array}$ & $\begin{array}{l}(23.7- \\
38.3)\end{array}$ & $\begin{array}{l}(45.7- \\
50.0)\end{array}$ \\
\hline \multirow[t]{2}{*}{60 years old or more } & 64.5 & 48.8 & 31.6 & 10.7 & 51.0 & 35.0 \\
\hline & $\begin{array}{l}(59.6- \\
69.2)\end{array}$ & $\begin{array}{l}(44.9- \\
52.7)\end{array}$ & $\begin{array}{l}(29.4- \\
33.9)\end{array}$ & $(8.4-13.5)$ & $\begin{array}{l}(43.0- \\
58.9)\end{array}$ & $\begin{array}{l}(32.9- \\
37.1)\end{array}$ \\
\hline \multicolumn{7}{|l|}{ Region of residence } \\
\hline \multirow[t]{2}{*}{ Southeast } & 42.7 & 37.1 & 36.6 & 45.3 & 48.4 & 38.9 \\
\hline & $\begin{array}{l}(39.8- \\
45.7)\end{array}$ & $\begin{array}{l}(34.8- \\
39.6)\end{array}$ & $\begin{array}{l}(34.9- \\
38.2)\end{array}$ & $\begin{array}{l}(42.5- \\
48.1)\end{array}$ & $\begin{array}{l}(45.8- \\
51.1)\end{array}$ & $\begin{array}{l}(37.3- \\
40.6)\end{array}$ \\
\hline
\end{tabular}

Total $^{1}$ : individuals with at least one of the selected diseases and limitations due to one of these diseases. Race: obtained by self-classification according to the IBGE categories; later categorized as white and nonwhite (black, brown, yellow, or indigenous) ${ }^{2}$. Education: categorized into incomplete elementary school (no education or incomplete elementary school), high school (complete high school and incomplete college) and higher education (complete college or more) ${ }^{3}$. Social score: classification proposed by the Brazilian Association of Population Studies (http://www.abep.org/criterio-brasil), where the population is divided into five categories, ranging from $A$ (richest) to $E$ (poorest) ${ }^{4}$. 


\begin{tabular}{|c|c|c|c|c|c|c|}
\hline \multirow[t]{3}{*}{ Variables } & CVA & Arthritis & Back pain & WMSD & COPD & Total $^{1}$ \\
\hline & $\%$ & $\%$ & $\%$ & $\%$ & $\%$ & $\%$ \\
\hline & (Cl 95\%) & (Cl 95\%) & (Cl 95\%) & (Cl 95\%) & (Cl 95\%) & (Cl 95\%) \\
\hline \multirow[t]{2}{*}{ South } & 14.0 & 18.3 & 19.8 & 24.9 & 28.7 & 18.8 \\
\hline & $\begin{array}{l}(12.2- \\
16.0)\end{array}$ & $\begin{array}{l}(16.8- \\
20.0)\end{array}$ & $\begin{array}{l}(18.5- \\
21.1)\end{array}$ & $\begin{array}{l}(22.2- \\
27.7)\end{array}$ & $\begin{array}{l}(26.3- \\
31.3)\end{array}$ & $\begin{array}{l}(17.7- \\
20.1)\end{array}$ \\
\hline \multirow[t]{2}{*}{ Midwest } & 5.8 & 7.8 & 7.6 & 9.1 & 7.0 & 7.7 \\
\hline & $(5.0-6.7)$ & $(6.8-8.8)$ & $(7.0-8.4)$ & $(7.3-11.3)$ & $(6.2-7.8)$ & $(7.1-8.3)$ \\
\hline \multirow[t]{2}{*}{ Northeast } & 29.8 & 30.9 & 30.3 & 18.4 & 11.8 & 29.0 \\
\hline & $\begin{array}{l}(27.7- \\
32.0)\end{array}$ & $\begin{array}{l}(29.0- \\
32.9)\end{array}$ & $\begin{array}{l}(28.8- \\
31.9)\end{array}$ & $\begin{array}{l}(16.8- \\
20.1)\end{array}$ & $\begin{array}{l}(10.9- \\
12.9)\end{array}$ & $\begin{array}{l}(27.6- \\
30.4)\end{array}$ \\
\hline \multirow[t]{2}{*}{ North } & 7.7 & 5.9 & 5.7 & 2.3 & 4.0 & 5.6 \\
\hline & $(7.0-8.5)$ & $(5.4-6.4)$ & $(5.2-6.3)$ & $(2.1-2.6)$ & $(3.6-4.4)$ & $(5.2-6.1)$ \\
\hline \multicolumn{7}{|c|}{$\begin{array}{l}\text { Total }{ }^{1} \text { : individuals with at least one of the selected diseases and limitations due to one of these diseases. } \\
\text { Race: obtained by self-classification according to the IBGE categories; later categorized as white and non- } \\
\text { white (black, brown, yellow, or indigenous) }{ }^{2} \text {. Education: categorized into incomplete elementary school (nc } \\
\text { education or incomplete elementary school), high school (complete high school and incomplete college) } \\
\text { and higher education (complete college or more) }{ }^{3} \text {. Social score: classification proposed by the Brazilian } \\
\text { Association of Population Studies (http://www.abep.org/criterio-brasil), where the population is divided } \\
\text { into five categories, ranging from A (richest) to E (poorest) }{ }^{4} \text {. }\end{array}$} \\
\hline
\end{tabular}

In Table 4, the prevalence of use of rehabilitation therapies was higher among the richest, among women, those who reported white race, higher education or more, aged between 40 and 59 years, and living in the South Region. For COPD, the prevalence of rehabilitation therapies was higher among men, non-whites, with higher education or more, higher social score, and living in the North and Midwest regions. Regarding WMSD, the prevalence of use of rehabilitation therapy was higher among individuals, non-white races, and residents of the Northeast region. 
Table 4

Prevalence of rehabilitation therapies among adult population with chronic diseases who reported moderate, intense, or very intense limitations in daily activities by sociodemographic characteristics. PNS, Brazil, 2013.

\begin{tabular}{|c|c|c|c|c|c|c|}
\hline \multirow[t]{4}{*}{ Variables } & CVA & Arthritis & Back pain & WMSD & COPD & Total $^{1}$ \\
\hline & Therapy + & Therapy+ & Therapy+ & Therapy+ & Therapy+ & Therapy+ \\
\hline & & $\%$ & $\%$ & $\%$ & $\%$ & $\%$ \\
\hline & $\begin{array}{l}\% \\
\text { (Cl 95\%) }\end{array}$ & (Cl 95\%) & (Cl 95\%) & (IC 95\%) & (IC 95\%) & (IC 95\%) \\
\hline
\end{tabular}

\section{Sex}

Men

27,6

30,5

16.5

31.5

11.2

21.3

(17.6-4.5)

(23.6-

(13.8-

19.6)

(23.1-

41.3)

(9.7-12.8)

(18.4-

38.3)

22.1

34.8

9.0

24.6)

Women

33.3

38.4

(34.0-

43.0)

(19.5-

24.8)

(29.1-

41.1)

(6.7-12.0)

29.2

41.9)

\section{Race}

White

40.5

36.2

23.2

29.8

9.3

29.3

(33.3-

(30.9-

41.8)

(20.1-

26.6)

(23.6-

36.9)

(7.1-12.2)

(26.4-

32.5)

Non-White

20.9
$(17.3-$
$25.0)$

36.8

17.0

37.7

10.8

23.5

(31.6-

(14.6-

19.7)

(30.0-

46.1)

(8.7-13.3)

(21.126.0)

\section{Education}

Elementary

incomplete

\begin{tabular}{lllllll|} 
& $(21.4-$ & $(32.0-$ & $(14.5-$ & $(27.4-$ & $(7.9-12.1)$ & $(20.4-$ \\
& $29.2)$ & $40.8)$ & $19.6)$ & $37.7)$ & & $25.2)$ \\
\hline High School & 34.5 & 34.6 & 22.1 & 33.6 & 9.9 & 29.1 \\
\hline & $(18.6-$ & $(28.1-$ & $(18.6-$ & $(24.2-$ & $(6.9-14.0)$ & $(25.6-$ \\
Higher education & $54.9)$ & $41.8)$ & $25.9)$ & $44.5)$ & & $32.9)$ \\
& 49.5 & 42.5 & 33.9 & 43.1 & 11.1 & 39.1 \\
& $(25.2-$ & $(28.7-$ & $(27.0-$ & $(31.3-$ & $(7.1-17.0)$ & $(32.8-$ \\
& $74.0)$ & $57.6)$ & $41.6)$ & $55.6)$ & & $45.7)$ \\
\hline
\end{tabular}

Total: individuals with at least one of the selected diseases and limitations due to one of these diseases ${ }^{1}$. Therapy +: prevalence of individuals who reported use of one or more rehabilitation therapies ${ }^{2}$. 


\begin{tabular}{|c|c|c|c|c|c|c|}
\hline \multirow[t]{2}{*}{ Variables } & CVA & Arthritis & Back pain & WMSD & COPD & Total $^{1}$ \\
\hline & $\begin{array}{l}\text { Therapy + } \\
2 \\
\% \\
\text { (Cl 95\%) }\end{array}$ & $\begin{array}{l}\text { Therapy+ } \\
\% \\
\text { (Cl 95\%) }\end{array}$ & $\begin{array}{l}\text { Therapy+ } \\
\% \\
\text { (Cl 95\%) }\end{array}$ & $\begin{array}{l}\text { Therapy+ } \\
\% \\
\text { (IC 95\%) }\end{array}$ & $\begin{array}{l}\text { Therapy+ } \\
\% \\
\text { (IC 95\%) }\end{array}$ & $\begin{array}{l}\text { Therapy+ } \\
\% \\
\text { (IC 95\%) }\end{array}$ \\
\hline \multicolumn{7}{|l|}{ Social Score } \\
\hline \multirow[t]{2}{*}{$A+B$} & 40.6 & 42.6 & 30.4 & 35.0 & 13.1 & 34.5 \\
\hline & $\begin{array}{l}(25.7- \\
57.5)\end{array}$ & $\begin{array}{l}(33.2- \\
52.6)\end{array}$ & $\begin{array}{l}(25.5- \\
35.8)\end{array}$ & $\begin{array}{l}(24.4- \\
47.3)\end{array}$ & $(9.9-17.2)$ & $\begin{array}{l}(30.2- \\
39.0)\end{array}$ \\
\hline \multirow[t]{2}{*}{ C } & 28.4 & 38.6 & 20.4 & 34.8 & 6.4 & 28.0 \\
\hline & $\begin{array}{l}(21.1- \\
37.0)\end{array}$ & $\begin{array}{l}(33.3- \\
44.1)\end{array}$ & $\begin{array}{l}(17.2- \\
23.9)\end{array}$ & $\begin{array}{l}(29.0- \\
41.1)\end{array}$ & $(4.1-9.8)$ & $\begin{array}{l}(24.9- \\
31.3)\end{array}$ \\
\hline \multirow[t]{2}{*}{$D+E$} & 28.0 & 29.6 & 13.1 & 29.6 & 10.7 & 18.6 \\
\hline & $\begin{array}{l}(22.9- \\
33.7)\end{array}$ & $\begin{array}{l}(24.3- \\
35.5)\end{array}$ & $\begin{array}{l}(10.8- \\
15.8)\end{array}$ & $\begin{array}{l}(21.2- \\
39.6)\end{array}$ & $(9.5-12.1)$ & $\begin{array}{l}(16.1- \\
21.4)\end{array}$ \\
\hline \multicolumn{7}{|l|}{ Age group } \\
\hline \multirow[t]{2}{*}{18 to 39 years old } & 19.9 & 27.9 & 16.2 & 19.3 & 4.9 & 17.7 \\
\hline & $(7.7-42.6)$ & $\begin{array}{l}(20.3- \\
37.0)\end{array}$ & $\begin{array}{l}(12.7- \\
20.5)\end{array}$ & $\begin{array}{l}(12.5- \\
28.6)\end{array}$ & $(3.3-7.4)$ & $\begin{array}{l}(14.5- \\
21.5)\end{array}$ \\
\hline \multirow[t]{2}{*}{40 to 59 years old } & 23.5 & 40.5 & 21.1 & 41.4 & 13.8 & 28.8 \\
\hline & $\begin{array}{l}(17.5- \\
30.7)\end{array}$ & $\begin{array}{l}(34.9- \\
46.3)\end{array}$ & $\begin{array}{l}(18.2- \\
24.3)\end{array}$ & $\begin{array}{l}(34.5- \\
48.6)\end{array}$ & $\begin{array}{l}(10.3- \\
18.1)\end{array}$ & $(26.0-31.9)$ \\
\hline \multirow[t]{2}{*}{60 years old or more } & 34.0 & 33.8 & 20.3 & 26.9 & 9.6 & 26.9 \\
\hline & $\begin{array}{l}(28.4- \\
40.0)\end{array}$ & $\begin{array}{l}(28.8- \\
39.3)\end{array}$ & $\begin{array}{l}(17.2- \\
23.7)^{-}\end{array}$ & $\begin{array}{l}(17.1- \\
39.7)\end{array}$ & $(7.2-12.7)$ & $\begin{array}{l}(23.7- \\
30.5)\end{array}$ \\
\hline \multicolumn{7}{|l|}{ Region of residence } \\
\hline \multirow[t]{2}{*}{ Southeast } & 31.7 & 36.4 & 20.7 & 34.3 & 10.6 & 26.5 \\
\hline & $\begin{array}{l}(24.3- \\
40.1)\end{array}$ & $\begin{array}{l}(29.3- \\
44.2)\end{array}$ & $\begin{array}{l}\left(17.2^{-}\right. \\
24.7)\end{array}$ & $\begin{array}{l}(26.5- \\
43.0)\end{array}$ & $(8.1-13.8)$ & $\begin{array}{l}(23.1- \\
30.1)\end{array}$ \\
\hline \multirow[t]{2}{*}{ South } & 41.8 & 43.3 & 26.9 & 31.4 & 7.8 & 32.1 \\
\hline & $\begin{array}{l}(31.1- \\
53.4)\end{array}$ & $\begin{array}{l}(35.6- \\
51.3)\end{array}$ & $\begin{array}{l}(21.7- \\
32.8)\end{array}$ & $\begin{array}{l}(22.7- \\
41.6)\end{array}$ & $(6.4-9.4)$ & $\begin{array}{l}(27.6- \\
37.0)\end{array}$ \\
\hline
\end{tabular}

Total: individuals with at least one of the selected diseases and limitations due to one of these diseases ${ }^{1}$. Therapy +: prevalence of individuals who reported use of one or more rehabilitation therapies ${ }^{2}$. 


\begin{tabular}{|c|c|c|c|c|c|c|}
\hline \multirow[t]{2}{*}{ Variables } & CVA & Arthritis & Back pain & WMSD & COPD & Total $^{1}$ \\
\hline & $\begin{array}{l}\text { Therapy + } \\
2 \\
\% \\
\text { (Cl 95\%) }\end{array}$ & $\begin{array}{l}\text { Therapy+ } \\
\% \\
\text { (Cl 95\%) }\end{array}$ & $\begin{array}{l}\text { Therapy+ } \\
\% \\
\text { (Cl 95\%) }\end{array}$ & $\begin{array}{l}\text { Therapy+ } \\
\% \\
\text { (IC 95\%) }\end{array}$ & $\begin{array}{l}\text { Therapy+ } \\
\% \\
\text { (IC 95\%) }\end{array}$ & $\begin{array}{l}\text { Therapy+ } \\
\% \\
\text { (IC 95\%) }\end{array}$ \\
\hline \multirow[t]{2}{*}{ Midwest } & 33.4 & 28.6 & 24.7 & 34.0 & 16.0 & 28.6 \\
\hline & $\begin{array}{l}(23.6- \\
44.9)\end{array}$ & $\begin{array}{l}(21.9- \\
36.4\end{array}$ & $\begin{array}{l}(20.3- \\
29.8)\end{array}$ & $\begin{array}{l}(21.8- \\
48.8)\end{array}$ & $\begin{array}{l}(14.4- \\
17.8)\end{array}$ & $\begin{array}{l}(24.6- \\
33.1)\end{array}$ \\
\hline \multirow[t]{2}{*}{ Northeast } & 21.1 & 35.5 & 14.1 & 38.5 & 4.2 & 22.1 \\
\hline & $\begin{array}{l}(16.4- \\
26.6)\end{array}$ & $\begin{array}{l}(29.6- \\
41.7)\end{array}$ & $\begin{array}{l}(11.7- \\
16.9)\end{array}$ & $\begin{array}{l}(31.5- \\
46.0)\end{array}$ & $(3.9-4.5)$ & $\begin{array}{l}(19.6- \\
25.0)\end{array}$ \\
\hline \multirow[t]{2}{*}{ North } & 33.7 & 31.1 & 15.9 & 20.0 & 24.6 & 23.3 \\
\hline & $\begin{array}{l}(27.8- \\
40.0)\end{array}$ & $\begin{array}{l}(23.3- \\
40.0)\end{array}$ & $\begin{array}{l}(11.7- \\
21.1)\end{array}$ & $\begin{array}{l}(11.5- \\
32.4)\end{array}$ & $\begin{array}{l}(17.7- \\
33.2)\end{array}$ & $\begin{array}{l}(19.3- \\
27.9)\end{array}$ \\
\hline
\end{tabular}

In Table 5, female individuals with higher education or more, wealthier ( $A+B$ classes), between 40 and 59 years old, and residing in the South Region had a significantly higher chance (AOR) of using therapies for rehabilitation when compared with the other categories. Regarding back pain, wealthier women with higher education, belonging to wealthier classes ( $A+B$ classes), aged 60 years or more, and residing in the South Region had a higher chance of using rehabilitation therapies. White individuals and those living in the North region, with a self-reported diagnosis of stroke, had a significantly higher chance of using rehabilitation therapies when compared to the reference categories. Except for stroke, the race variable was not statistically significant when compared to its reference category. Regarding COPD, the age group from 40 to 59 years old and those living in the North and Midwest regions had a significantly greater chance of using rehabilitation therapies when compared to the other reference categories. 
Table 5

Chance of use of rehabilitation therapies for chronic diseases that caused moderate, intense, or very intense limitations in daily activities among adult population by sociodemographic characteristics. PNS, Brazil, 2013.

\begin{tabular}{lllllll}
\hline Variables & CVA & Arthritis & Back pain & WMSD & COPD & Total $^{1}$ \\
\cline { 2 - 5 } & AOR & AOR & AOR & AOR & AOR & AOR \\
\hline Sex & 1 & 1 & 1 & 1 & 1 & 1 \\
\hline Men & 1.6 & 1.4 & 1.3 & 1.1 & 0.7 & 1.5 \\
\hline Women & $(0.8-3.3)$ & $(1.0-2.1)$ & $(1.0-1.7)^{*}$ & $(0.7-1.9)$ & $(0.4-1.1)$ & $(1.2-1.8)$ \\
\hline Race & & & & & & \\
\hline Non-white & 1 & 1 & 1 & 1 & 1 & 1 \\
\hline White & 2.1 & 0.8 & 1.0 & 0.7 & 1.0 & 1.1 \\
\hline & $(1.1-4.1)$ & $(0.6-1.1)$ & $(0.8-1.4)$ & $(0.4-1.2)$ & $(0.5-1.8)$ & $(0.9-1.4)$ \\
\hline
\end{tabular}

\section{Education}

\begin{tabular}{lllllll} 
Elementary incomplete & 1 & 1 & 1 & 1 & 1 & 1 \\
\hline High School & 1.8 & 0.8 & 1.2 & 1.1 & 0.8 & 1.2 \\
\hline Higher education & $(0.8-4.1)$ & $(0.5-1.1)$ & $(1.0-2.6)^{*}$ & $(0.6-1.9)$ & $(0.4-1.6)$ & $(0.9-1.6)$ \\
\hline & 2.7 & 0.9 & 2.1 & 2.4 & 1.1 & 1.7 \\
\hline
\end{tabular}

\section{Social Score}

$\begin{array}{lllllll}\mathrm{D}+\mathrm{E} & 1 & 1 & 1 & 1 & 1 & 1 \\ \mathrm{~A}+\mathrm{B} & 1.0 & 1.9 & 1.5 & 1.3 & 1.4 & 1.7 \\ & (0.4-2.9) & (1.1-3.3) & (1.1-2.0) & (0.5-3.2) & (0.8-2.5) & (1.2-2.5) \\ \mathrm{C} & 0.5 & 1.5 & 1.4 & 1.4 & 0.5 & 1.6 \\ & (0.3-1.1) & (1.1-2.1) & (1.0-2.0) * & (0.7-2.7) & (0.3-0.9) & (1.2-2.0)\end{array}$

\section{Age group}

\begin{tabular}{lllllll}
18 to 39 years old & 1 & 1 & 1 & 1 & 1 & 1 \\
\hline 40 to 59 years old & 1.2 & 1.6 & 1.4 & 3.7 & 4.2 & 2.1 \\
\hline & $(0.3-4.6)$ & $(1.0-2.6)$ & $(1.0-2.0) *$ & $(2.0-7.0)$ & $(2.0-8.8)$ & $(1.6-2.7)$
\end{tabular}

\footnotetext{
* statistically significant $(\mathrm{p}<0.05) ; \mathrm{AOR}^{1}$ : Adjusted odds ratio, obtained by logistic regression model, considering the complex design of the sample. Outcome: report of carrying out one or more rehabilitation therapies.
} 


\begin{tabular}{|c|c|c|c|c|c|c|}
\hline \multirow[t]{2}{*}{ Variables } & CVA & Arthritis & Back pain & WMSD & COPD & Total $^{1}$ \\
\hline & AOR $^{1}$ & AOR & AOR & AOR & AOR & AOR \\
\hline \multirow[t]{2}{*}{60 years old or more } & 1.9 & 1.2 & 1.5 & 1.7 & 2.3 & 2.0 \\
\hline & $(0.5-7.5)$ & $(0.7-2.0)$ & $(1.0-2.1)^{\star}$ & $(0.8-3.7)$ & $(1.3-4.3)$ & $(1.5-2.7)$ \\
\hline \multicolumn{7}{|l|}{ Region of residence } \\
\hline Northeast & 1 & 1 & 1 & 1 & 1 & 1 \\
\hline \multirow[t]{2}{*}{ Southeast } & 1.7 & 0.9 & 1.2 & 0.7 & 2.7 & 1.0 \\
\hline & $(1.1-2.6)$ & $(0.6-1.4)$ & $(0.9-1.6)$ & $(0.4-1.2)$ & $(1.9-3.9)$ & $(0.7-1.2)$ \\
\hline \multirow[t]{2}{*}{ South } & 2.2 & 1.3 & 1.8 & 0.8 & 1.8 & 1.4 \\
\hline & $(1.1-4.3)$ & $(0.9-2.1)$ & $(1.3-2.7)$ & $(0.4-1.4)$ & $(1.2-2.7)$ & $(1.0-1.8)^{*}$ \\
\hline \multirow[t]{2}{*}{ Midwest } & 2.1 & 0.7 & 1.7 & 0.8 & 5.2 & 1.2 \\
\hline & $(0.9-4.6)$ & $(0.4-1.0)$ & $(1.2-2.4)$ & $(0.3-1.9)$ & $(3.9-7.1)$ & $(0.9-1.6)$ \\
\hline \multirow[t]{2}{*}{ North } & 2.4 & 0.9 & 1.1 & 0.3 & 9.7 & 1.0 \\
\hline & $(1.4-4.1)$ & $(0.5-1.4)$ & $(0.7-1.7)$ & $(0.1-0.5)$ & $(5.6-16.9)$ & $(0.8-1.5)$ \\
\hline
\end{tabular}

\section{Discussion}

About a quarter of the Brazilian population was diagnosed with a stroke, arthritis, WMSD, COPD, or reported back pain, and one in three affected by these diseases and conditions had moderate, intense, or very intense limitations of their usual activities. However, about one in four Brazilians with the chronic diseases listed in this study and moderate to severe limitations in their usual activities reported using rehabilitation therapy for their conditions or diseases.

The scenario of relatively low use of rehabilitation therapies among people with chronic diseases and limited daily activities may indicate challenges in accessing rehabilitation services, including barriers on both the demand and the supply for these service ${ }^{\mathrm{11}}{ }^{12}$. Examples of barriers in demand are the high cost of accessing services, including transport costs, waiting time, and lack of awareness of rehabilitation needs. In terms of supply, the absence of an integrated national policy on rehabilitation, inadequate provision of services, insufficient infrastructure, lack of professionals and professionals with little training can be included ${ }^{11,12,13}$.

Although the PNS-2013 does not address the possible barriers to the use of rehabilitation therapies in the population studied, all individuals should be able to access quality rehabilitation services without fear of financial difficulties, according to the WHO. In Brazil, about $72 \%$ of the Brazilian population depends exclusively on the Unified Health System (SUS), and, despite social inequalities in access to medical and 
dental services, they are offered almost universally ${ }^{14,15}$. However, the offer of rehabilitation services through the Unified Health System does not have a consolidated policy, legislation, or even a specific budget ${ }^{13}$, having sparse regulations from different areas of the Ministry of Health, such as Occupational Health, Elderly Health, and Disability, Trauma and Violence Program ${ }^{13}$.

In Brazil, rehabilitation services are offered predominantly in urban areas, concentrated in the most economically developed regions, and with a low assistance coverage, mostly offered in a precarious network with little articulation with an integrative and multidisciplinary proposal ${ }^{16,17}$. The concentration of these services occurs in hospital care at the expense of primary health care, with few reference services and difficulties reported by patients related to distance, cost, transport, and accessibility, in addition to the delay in scheduling these services and the vulnerability of the lower-income population $17,18,19$. These barriers may explain the present study's findings, where we observed higher chances of using rehabilitation therapies among the female population, with higher education, higher socioeconomic status, older, and living in the Southern region of Brazil.

Although the clear benefits of rehabilitation therapies in the conditions and chronic diseases studied, this was not often reported by the subjects ${ }^{20,21}$. In the case of post-stroke patients, who had moderate to very severe limitations, less than $1 / 3$ were undergoing any therapy due to stroke, an even lower proportion (slightly more than $1 / 4$ ) when considering all the chronic diseases studied 22 .

Although stroke is less prevalent among the conditions studied, it has the highest percentage of individuals with moderate to very intense impairment. Schmidt et al. noted that the change in the epidemiological profile that has taken place in recent decades in Brazil has highlighted diseases of the circulatory system and, among the most important, stroke, one of the leading causes of hospitalization and mortality ${ }^{23}$. The literature indicates that most post-stroke patients have chronic sequelae that require rehabilitation to restore their functionality in their daily activities ${ }^{22,23}$. In this study, the percentage of people who reported medium, intense, or very intense degrees of limitations in activities of daily living was $38 \%$. Considering the disabling potential of stroke, the active monitoring of patients should occur from the onset of the disease, with a rehabilitation program aimed at joint protection, maintenance of the functional state of the locomotor system, and the cardiorespiratory system ${ }^{24}$.

In the present study, individuals who reported having WMSD were the ones who most frequently used rehabilitation therapies. WMSDs are damage resulting from overuse imposed on the musculoskeletal system, without adequate time for recovery ${ }^{26}$. According to a study developed by the Ministry of Health, WMSD affects $50 \%$ to $80 \%$ of the economically active population ${ }^{27}$. Age, gender, time working in the profession, and education may be factors associated with WMSD, considering economic activity and occupation. One study observed high proportions of symptoms related to WMSD, such as limit or inability to perform tasks, in addition to withdrawal from work activities ${ }^{28}$.

In this study, individuals who reported having COPD, aged between 40 and 59 years, were significantly more likely to use rehabilitation therapies when compared to the other reference categories. COPD is among the leading causes of morbidity and mortality in developing countries ${ }^{29}$. In Brazil, COPD is among the ten leading 
causes of death ${ }^{29}$. The specialized literature indicates that pulmonary rehabilitation improves exercise capacity, reduces dyspnea, and improves the overall quality of life, and its benefits surpass any other therapy. In addition, pulmonary rehabilitation increases the functional exercise capacity, reduces hospitalizations, and reduces the cost of treatment ${ }^{30,31}$.

The study's main limitations are related to the structure of the PNS-2013 questions, which limits the possible answers for the use of rehabilitation therapies to up to three options, at most, for each chronic disease. Another limitation of the questionnaire is related to the option "physical exercise" as rehabilitation therapy, sometimes offering the option "exercise or some physical activity" (in case the respondent has reported a stroke), sometimes offering the option "exercise or physiotherapy," combined, without the possibility of analyzing the two separately or distinguishing whether the practice was prescribed or monitored by a health professional.

For the present study, we decided to use any positive response (exercise or exercise and physiotherapy) to characterize the use of rehabilitation therapies, implying putting on the same level the use of physiotherapy, which depends on access to health professionals, and physical activity, which can be guided by a health professional or practiced spontaneously by the respondent. Future studies should separate possible rehabilitation therapies in their analysis.

The authors' option to analyze all chronic diseases and the resulting use or not of rehabilitation therapies together, without discriminating the factors that interfere in using these therapies for each chronic disease listed, can be considered a limitation as a fortress. The potential limitation stems from the fact that each disease has different consequences on the individual's health, with variations in the frequency and intensity of limitations in daily activities caused by morbidities. However, we consider this option an opportunity since rehabilitation therapies are necessary for moderate or severe limitations resulting from morbidities. The use of one or more therapies can be a marker of both access to health services and self-care in the case of physical activity.

We recommend studies to assess individual perceptions of the need for rehabilitation, access to and barriers to health services, factors that improve patient adherence to rehabilitation therapies, and the geographic distribution of these services.

\section{Conclusions}

The relatively low use of rehabilitation therapies among Brazilian adults with functional limitations resulting from chronic diseases, potentially benefiting from these therapies, highlights the need for universalization and expansion of the offer of this type of service, especially among the socially and economically more vulnerable population and more dependent on the Unified Health System. Adopting a national public policy that guarantees and articulates equity of access to rehabilitation therapies and health promotion actions that improve adherence to necessary treatments can improve this scenario in Brazil.

\section{Abbreviations}


WHO - World Health Organization

SUS - Sistema Único de Saúde - Unified Health System

PNS - Pesquisa Nacional de Saúde - National Health Survey

IBGE - Instituto Brasileiro de Geografia e Estatística - Brazilian Institute of Geography and Statistics

UPA - Primary Analysis Units

WMSD - Work-related Musculoskeletal Disorder

COPD - chronic obstructive pulmonary disease

OR - Odd Ratio

AOR - Ajusted Odd Ratio

$\mathrm{Cl}$ - Confidence Interval

\section{Declarations}

\section{Ethics approval and consent to participate}

The datasets used in this study were obtained from the open-data reports (https://www.pns.icict.fiocruz.br/). The Ministry of Health of Brazil is committed to respecting the ethical precepts and guaranteeing the privacy and reliability of the data. According to Brazilian laws, this study that uses public and anonymized secondary data is waved from ethical approval.

\section{Consent for Publication}

All authors of the manuscript have read and agreed to its content. We are accountable for all aspects of the accuracy and integrity of the manuscript. We consent to the terms of the BioMed Central Copyright and License Agreement.

\section{Competing Interest}

The authors have no competing interests as defined by $\mathrm{BMC}$, or other interests that might be perceived to influence the results and/or discussion reported in this paper.

\section{Author Contribution}

PMMB \& CSB wrote the main manuscript text and prepared tables; CMRD, contribute with the analysis and all authors reviewed the manuscript.

\section{Acknowledgements}

Not applicable. 


\section{Funding}

No funding.

\section{Availability of Data and Materials}

The datasets generated and/or analysed during the current study are available in the PNS 2019 - Base de Dados Fiocruz, repository, https://www.pns.icict.fiocruz.br/bases-de-dados/.

\section{References}

1. Duarte Elisabeth Carmen, Barreto Sandhi Maria. Transição demográfica e epidemiológica: a Epidemiologia e Serviços de Saúde revisita e atualiza o tema. Epidemiol. Serv. Saúde. 2012;21(4): 529532.

2. GBD 2019 Diseases and Injuries Collaborators. Global burden of 369 diseases and injuries in 204 countries and territories, 1990-2019: a systematic analysis for the Global Burden of Disease Study 2019. Lancet. 2020;17;396(10258):1204-1222. Erratum in: Lancet. 2020;14;396(10262):1562.

3. Stucki G, Cieza A, Melvin J. The international classification of functioning, disability and health: a unifying model for the conceptual description of the rehabilitation strategy. J Rehabil Med. 2007;39(4):279-285.

4. Organização Mundial da Saúde (OMS). Relatório mundial sobre a deficiência/World Report on Disability. São Paulo: Secretaria dos Direitos da Pessoa com Deficiência; https://apps.who.int/iris/bitstream/handle/10665/44575/9788564047020_por.pdf (2012). Accessed 30 Apr 2021.

5. Caetano LA, Sampaio RF, Costa LA. A expansão dos serviços de reabilitação no SUS à luz do arcabouço normativo federal. Rev. Ter. Ocup. Univ. São Paulo. 2018;29(3):195-203.

6. Brasil. Ministério da Saúde. Secretaria de Atenção à Saúde. Departamento de Atenção Básica. Diretrizes para o cuidado das pessoas com doenças crônicas nas redes de atenção à saúde e nas linhas de cuidado prioritárias. Ministério da Saúde, Secretaria de Atenção à Saúde, Departamento de Atenção Básica. Brasília https://bvsms.saude.gov.br/bvs/publicacoes/diretrizes\%20_cuidado_pessoas\%20_doencas_cronicas.pdf (2013). Accessed 20 Apr 2021.

7. Souza-Junior PRB, Freitas MPS, Antonaci GA, Szwarcwald CL. Desenho da amostra da Pesquisa Nacional de Saúde 2013. Epidemiol Serv Saude 2015; 24(2):207-216.

8. Damacena GN, Szwarcwald CL, Malta DC, Souza-Junior PRB, Vieira MLFP, Pereira CA, Morais Neto OL, Silva Júnior JB. O processo de desenvolvimento da Pesquisa Nacional de Saúde no Brasil, 2013. Epidemiol Serv Saude 2015; 24(2):197-206.

9. IBGE, Instituto Brasileiro de Geografia e Estatística. PNS, Pesquisa Nacional de Saúde, 2013. Questionário dos moradores do domicílio. Diretoria de Pesquisas. Coordenação de Trabalho e Rendimento. https://ftp.ibge.gov.br/PNS/2013/Microdados/Documentacao/Questionario_PNS_2013.pdf (2013). Accessed 10 Apr 2021. 
10. ABEP, Associação Brasileira de Estudos Populacionais. Critério Brasil. http://www.abep.org/criterio-brasil (2016). Accessed 10 Apr 2020.

11. Jacobs B, Ir P, Bigdeli M, Annear PL, Van Damme W. Addressing access barriers to health services: an analytical framework for selecting appropriate interventions in low-income Asian countries. Health Policy Plan 2012;27(4):288-300.

12. Franchi EF, Piber VD, Selau CM, Schmidt MH, Soares P da S, Quatrin LB. Prevalência de pessoas com deficiência física e acesso ao serviço de reabilitação no Brasil. Cinergis. (2017);18(3):169.

13. Ribeiro CTM, Ribeiro MG, Araújo AP, Mello LR, Rubim LC, Ferreira JES. O sistema público de saúde e as ações de reabilitação no Brasil. Rev Panam Salud Publica. 2010;28(1):43-8.

14. Viacava F, Bellido JG. Health, access to services and sources of payment, according to household surveys. Cien Saude Colet. 2016;21(2):351-370.

15. Boccolini CS, de Souza Junior PR. Inequities in Healthcare utilization: results of the Brazilian National Health Survey, 2013. Int J Equity Health. 2016 Nov 17;15(1):150.

16. Melo, TR, Weinert, LVC, Hoshino, MS, Maldaner, TP. Perfil dos usuários de serviços de reabilitação no Sistema Único de Saúde. Revista da Universidade Vale do Rio Verde, Três Corações (2017);15(2): 65-77. DOI: http://dx.doi.org/10.5892/ruvrd.v15i2.3281.

17. Moretto, L. C; Longo, G. Z; Boing, A. F; Arruda, M. P. Prevalence of the use of physical therapy services among the urban adult population of Lages, Santa Catarina. Braz. J. Phys. Ther. (2009); 13(2): 130-135.

18. Rodes, Carolina Hart et al. 0 acesso e o fazer da reabilitação na Atenção Primária à Saúde. Fisioterapia e Pesquisa (2017); 24(1):74-82.

19. Sérvio TC, Britto RR, de Melo Ghisi GL, da Silva LP, Silva LDN, Lima MMO, Pereira DAG, Grace SL. Barriers to cardiac rehabilitation delivery in a low-resource setting from the perspective of healthcare administrators, rehabilitation providers, and cardiac patients. BMC Health Serv Res. (2019); 2;19(1):615.

20. Richardson CR, Franklin B, Moy ML, Jackson EA. Advances in rehabilitation for chronic diseases: improving health outcomes and function. BMJ. 2019; doi: 10.1136/bmj.I2191. PMID: 31208954.

21. Zziwa, S., Babikako, H., Kwesiga, D. et al. Prevalence and factors associated with utilization of rehabilitation services among people with physical disabilities in Kampala, Uganda. A descriptive cross sectional study. BMC Public Health. 2019; doi:10.1186/s12889-019-8076-3.

22. Ribeiro KSQS, Neves R da F, Brito GEG, Sousa K de M, Lucena EM de F, Batista HRL. Acesso à Reabilitação no pós-AVC na cidade de João Pessoa, Paraíba. Rev Baiana Saúde Pública. (2012);36(3):699-712.

23. Schmidt, MH; Selau, CM; Soares, PS; Franchi, EF; Piber, VD; Quatrin, LB. Acidente Vascular Cerebral E Diferentes Limitações: Uma Análise Interdisciplinar. Arq Ciências da Saúde da UNIPAR. (2019);23(2):139-44.

24. Lucena EMDF, Mendes LM. A funcionalidade de usuários acometidos por AVE em conformidade com a acessibilidade à reabilitação. Acta Fisiatr. 2011;18(3):112-8.

25. Laurindo IMM, Ximenes AC, Lima FAC, Pinheiro GRC, Batistella LR, Bertolo MB, et al. Artrite reumatóide: Diagnóstico e Tratamento. Rev Bras Reumatol. (2004);44(6):435-42. 
26. BRASIL. Ministério da Saúde. Dor relacionada ao trabalho [Internet]. Brasilia: Editora Ministério da Saúde. 2012. 1-70 p. Disponível em: http://portalms.saude.gov.br/vigilancia-em-saude/publicacoes

27. Brasil. Ministério da Saúde. Secretaria de Vigilância em Saúde. Departamento de Vigilância de Doenças e Agravos não Transmissíveis e Promoção da Saúde. Saúde Brasil 2018 uma análise de situação de saúde e das doenças e agravos crônicos: desafios e perspectivas. 2019. 424 p.

28. Viegas LRT, Almeida MMC de. Perfil epidemiológico dos casos de LER/DORT entre trabalhadores da indústria no Brasil no período de 2007 a 2013. Rev Bras Saúde Ocup. 2016;41(0):1-10.

29. Cruz MM, Pereira M. Epidemiology of chronic obstructive pulmonary disease in Brazil: A systematic review and meta-analysis. Cienc e Saude Coletiva. 2020;25(11):4547-57.

30. Fernandes ABS. Reabilitação respiratória em DPOC - a importância da abordagem fisioterapêutica. Pulmão RJ - Atualizações Temáticas. 2009;1(1):71-8.

31. Wehrmeister FC, Knorst M, Jardim JR, Macedo SEC, Noal RB, Martínez-Mesa J, et al. Programas de reabilitação pulmonar em pacientes com DPOC. J Bras Pneumol. 2011;37(4):544-55. 